ARTICLE

\title{
An approach to the developmental and cognitive profile of the child with spina bifida
}

\author{
N Ramsundhar, MB ChB, FCPaed (SA); K Donald, MB ChB, MPhil, FCPaed (SA), MRCPCH (UK), Cert Paed Neurology (SA) \\ Division of Developmental Paediatrics, University of Cape Town and Spina Bifida Clinic, Red Cross War Memorial Children's Hospital, Cape Town, \\ South Africa
}

Corresponding author: K Donald (kirsty.donald@uct.ac.za)

\begin{abstract}
Myelomeningocele, or open spina bifida (SBM), is the most common congenital defect of the central nervous system. The brain anomalies described in SBM are varied and contribute to the complex phenotypic outcomes in neurocognition and behaviour. Several factors have an impact on the severity and type of cognitive outcome. The strongest association with cognitive dysfunction is the presence of hydrocephalus and its complications. Hydrocephalus occurs in $80-95 \%$ of cases and typically results in attenuation of cerebral white matter, particularly in the posterior aspects of the brain, which is important in the development of spatial skills.
\end{abstract}

S Afr Med J 2014;104(3):221. DOI:10.7196/SAMJ.8048

Myelomeningocele, or open spina bifida (SBM), is the most common congenital defect of the central nervous system. It is caused by failure of primary neurulation and, although the spinal abnormalities and their consequences are well known, it is also associated with a wide range of developmental abnormalities of the brain, resulting in a complex neurodevelopmental disorder with great phenotypic heterogeneity. However, the cognitive deficits in spina bifida are less well characterised and tend to be under-recognised, especially in resource-limited environments. They may have a significant impact on the lives of children with this disorder.

\section{Brain findings}

The brain anomalies described in SBM are varied and contribute to the complex phenotypic outcomes in neurocognition and behaviour. ${ }^{[1,2]}$ It is worth emphasising that closed forms of spina bifida seldom have brain anomalies.

Several factors have an impact on the severity and type of cognitive outcome. The strongest association with cognitive dysfunction is the presence of hydrocephalus and its complications. Hydrocephalus occurs in $80-95 \%$ of cases and typically results in attenuation of cerebral white matter, particularly in the posterior aspects of the brain, which is important in the development of spatial skills. Furthermore, complications of hydrocephalus treatment, such as shunt obstruction or shunt infection with ventriculitis, may have a dramatic impact on neurocognitive outcome.

The Chiari II malformation is invariably present in patients with a myelomeningocele. Although the main focus is on the malformation of the hindbrain and the cerebellum, it is a pancerebral malformation affecting the entire central nervous system. ${ }^{[3]}$ The cerebellum is responsible for fine motor co-ordination and has a critical role in specific cognitive functions such as attention, planning and learning. Partial dysgenesis of the corpus callosum is another common associated abnormality in SBM (Fig. 1)..$^{[1,4,5]}$

Additional factors that have a role in determining the longterm neurocognitive outcome include epilepsy (and its treatment), endocrine complications and the impact of other medical complications, which may result in repeated hospitalisations.

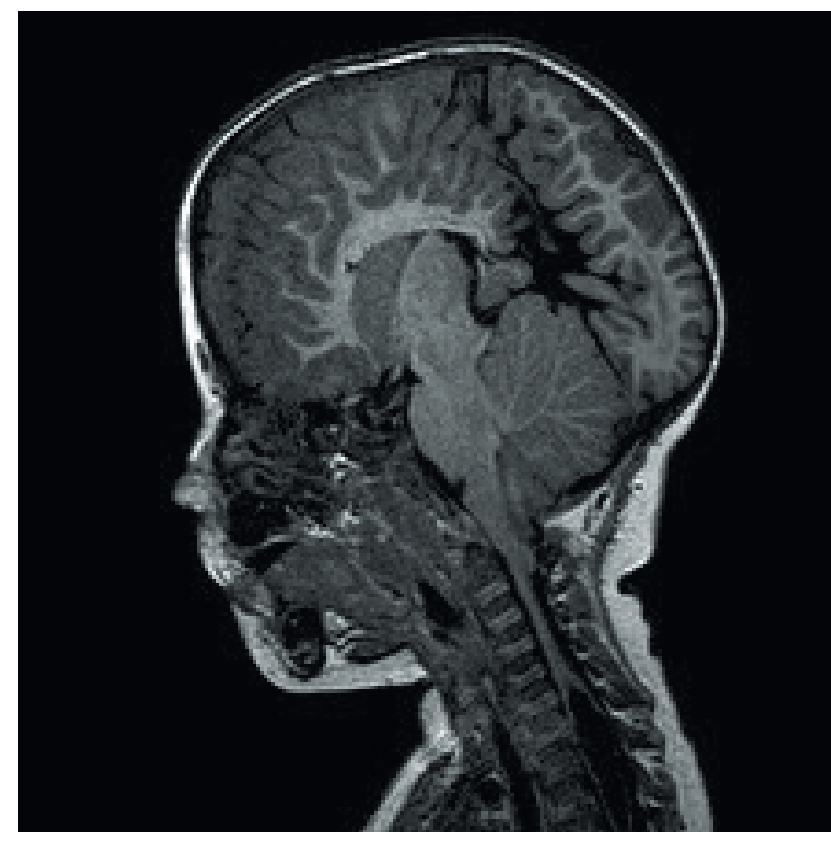

Fig. 1. Sagittal magnetic resonance imaging scan of the brain of a child with myelomeningocele, showing the shallow, crowded posterior fossa typical of a Chiari II malformation.

Furthermore, children with spina bifida from low socio-economic environments are at higher risk of poor cognitive outcomes, and high spinal cord lesions (above the level of T12) correlate inversely with cognitive ability. These factors are summarised in Table 1.

\section{Cognitive profile}

The cognitive profile of individuals with spina bifida is characterised by a typical pattern, i.e. the 'modal profile. ${ }^{[5]}$ Children with spina bifida generally display better scores in verbal intelligence quotient (IQ) than performance IQ. Specific performance IQ deficits include weaknesses in motor, visual-spatial and mathematical tasks. Total scores tend to be less useful than more specific subscales as there are 
Table 1. Risk factors for cognitive dysfunction in myelomeningocele $e^{[2,8,9]}$

Hydrocephalus

Complications related to hydrocephalus (the need for ventriculoperitoneal shunt; number of shunt revisions; encephalitis and seizures)

Additional structural abnormalities of the central nervous system such as Chiari II malformation

Level of spinal cord lesion

Endocrine complications

Recurrent hospitalisations for related medical complications (such as urinary tract infections)

Poor socio-economic status

Ethnicity

Table 2. Neurocognitive profile of children with spina bifida ${ }^{[7]}$

\begin{tabular}{lll}
\hline & Modal profile of children with spina bifida \\
\hline Domain & Strength & Weakness \\
\hline Intelligence & Verbal IQ & Performance IQ \\
Language & Lexical, syntactic & Discourse-level pragmatics \\
Memory & Rote & Reconstructive \\
Mathematics & Fact retrieval & Procedures \\
Attention & & Focused attention \\
Spatial analysis & Matching to sample & Orientation \\
Reading & Decoding & Comprehension
\end{tabular}

frequently significant discrepancies, even within domains. ${ }^{[5,6]}$ These are summarised in Table 2. ${ }^{[7]}$

\section{Intelligence}

The majority of individuals with spina bifida have an IQ in the average to low-average range. ${ }^{[8]}$ An Australian study found that $18.8 \%$ of individuals in their cohort had intellectual disability (IQ <70). ${ }^{[9]}$ In addition, children with spina bifida have an increased risk of cognitive and specific learning disorders, with $60 \%$ reported as having specific learning disorders. ${ }^{[10]}$ The most common types of learning disorder in the general population are language-related, such as dyslexia. The learning disorders of children with spina bifida are predominantly in the non-verbal or performance domains. ${ }^{[6]}$

\section{Language ability}

In the language domain, the development of basic vocabulary and grammar typically occurs in line with age-matched peers. ${ }^{[7]}$ However, children with spina bifida frequently demonstrate impairments in comprehension and the correct contextual use of language. The hyperverbal or 'cocktail party syndrome' is well described in these children. It is characterised by incessant talking that is superficially fluent, but the content often lacks complexity and may be inappropriate. Such children may also use stereotypies or occasionally echolalia to make their speech seem fluent.

The majority read well (good word recognition and fluency), but lack the ability to appropriately analyse or explain the text. Individuals with visual spatial weaknesses may experience further difficulties in reading, including skipping lines and reversing or misreading letters or numbers. ${ }^{[10,11]}$

\section{Memory and mathematical ability}

Children with spina bifida are reported to have relative strength in rote learning, which may mask problems in the more complex tasks of constructive memory. Rote learning is the ability to learn and recall information that has been learnt by association or repetition (e.g. lists, songs and numbers). This may occur with little understanding of the words or concept of numbers. They tend to display poor ability to apply information to situations different from the original learning situation. ${ }^{[6,10,11]}$ For example, children with SBM may correctly recite the $12 \times$ table. However, if asked for $12 \times 8$, they may start at $12 \times 1$, progressing in a sequential order to arrive at the answer for $12 \times 8$. They also have problems with long division, mathematical word sum problems and application of algorithms. ${ }^{[6]}$

\section{Executive function}

Executive function refers to a group of abilities responsible for goaldirected cognitive, behavioural and emotional functioning. Children with SBM tend to perform poorly in tests of executive function, although the specific profile varies between reported studies. ${ }^{[6,12]}$

\section{Attention}

Approximately a quarter of children with SBM are reported to have difficulties with attention. ${ }^{[6,8]}$ There are two attention networks - the anterior network for sustained attention and the posterior network for focus and shifting of attention. These children have deficits in the latter. This attention profile differs from the phenotype of children with attention deficit hyperactivity disorder, who tend to display anterior network deficiencies. ${ }^{[12]}$ Children with SBM respond poorly to stimulant therapy such as methylphenidate, possibly as a result of the different pathophysiology. ${ }^{[5,6]}$

\section{Visual-spatial deficits}

In normal physiology, visual motor processing skills result in the use of vision to co-ordinate the movement of body parts. Intact functioning of these skills is essential for tasks such as copying figures correctly or smooth synchronised movement. Visual discrimination is the process by which sight is used to distinguish one item from another. Visual sequencing is the ability to see and differentiate the order of symbols, words, or images.

The visual processing skills mentioned above are reliant on two pathways - the ventral and dorsal pathways. The former is responsible for object-based discrimination of shape, size, orientation, and recognition of faces. The latter is the action-based system responsible for spatial relations and the coupling of these co-ordinates to movement. In spina bifida, the dorsal visual processing pathway is impaired. The weaknesses of visual perception are evident in tasks 
such as drawing, route finding, route planning and visual pursuit. ${ }^{[6,13]}$ Furthermore, children with SBM (especially those with high spinal cord lesions) may have impairment of upper limb motor function, which is necessary to execute visually guided hand movements required in tasks such as drawing and copying a figure. They write more slowly, the letters/words are poorly spaced, and the letter formation is poor. Since most examinations are in written format, this deficit may have a significant impact on scholarly performance. ${ }^{[1]}$

\section{Approach to cognitive management}

The early identification of children with SBM who have developmental problems (especially those at increased risk of poor cognitive outcome) is important to allow early appropriate intervention and support. The optimal care of a child with spina bifida is complex and involves a multidisciplinary team approach. Ideally, there should be a co-ordinating non-specialist general practitioner or paediatrician who is best placed as the first point of contact with the child and family. Additional specialists who are frequently involved in optimising care and outcome include a developmental paediatrician, a neurologist, a neurosurgeon, an orthopaedic surgeon, and a renal team where necessary. ${ }^{[14,15]}$

In terms of developmental assessment, initial management includes a detailed history from the parents regarding the pregnancy, birth process, early developmental milestone attainment and developmental trajectory the child has followed to date. This is also an opportunity to establish parents' concerns about the child's cognitive ability and potential learning disorders.

Standardised developmental screening assessments and more formal tools should ideally be used early to determine the child's level of functioning, weaknesses and strengths. Depending on the strengths and weaknesses, the child is referred to a physiotherapist, a speech therapist and an occupational therapist for rehabilitation to improve mobilisation, quality of speech and comprehension, and visual-motor deficits, respectively. School planning can begin at this stage.

Although there are many therapeutic interventions, there is currently no evidence-based approved therapy for children with learning disabilities in the performance domain. There should be regular developmental review to monitor the response to therapies and ensure optimal health and identification of associated secondary complications. The prevention of complications such as urinary tract infections and further neurological insults will reduce hospitalisations, which are associated with a negative impact on development. Even in the absence of hydrocephalus, it is recommended that children with SBM should have at least one formal neuro-imaging study to exclude other associated brain abnormalities. Correctable causes of developmental delays such as hearing and visual impairment should always be ruled out. ${ }^{[13-15]}$

Ideally, a developmental paediatrician should monitor the child's neurobehavioural and developmental progress and assist in determining their suitability for mainstream or special education. In South Africa, best placement of the child generally requires early liaison with the regional educational psychologist serving the local department of education. Together they will determine the most appropriate special education facility or mainstream school that best meets the needs of the individual child.

Special considerations in recommending a school for a child with spina bifida include:
- accessibility for children with motor deficits, i.e. wheelchair access

- toilet facilities and school staff who can assist the child with clean intermittent catheterisation

- smaller or unit classes for children with SBM who have cognitive or learning difficulties.

The teacher needs to be made aware of the child's needs and an individual education plan can be tailored to enable the child to reach his/her full potential.

Although an inclusive education policy is in place in South Africa, there are very few public sector mainstream schools that can accommodate children who require any significant degree of medical or physical support. Furthermore, the number and accessibility of schools with facilities to accommodate children with special needs, be these motor deficits or learning difficulties, are limited, especially outside the major urban centres. Children with SBM are therefore often inadequately supported in a poorly resourced mainstream classroom environment. More severely physically disabled children may be kept out of school entirely if they are not able to access special schooling.

\section{Conclusion}

The developmental and cognitive sequelae of SBM, even though differing in scope and severity for individual children, may have a profound impact on their lives. The role of the developmental and rehabilitation team in identifying and supporting the developmental problems of children with SBM, as well as active planning and advocacy for their schooling options, are critical elements in facilitating their optimal outcome.

\section{References}

1. Vinck A, Maassen BAM, Mullaart R, Rotteveel JJ. Arnold-Chiari-2 malformation and cognitive functioning in spina bifida. J Neurol Neurosurg Psychiatry 2006;77:1083-1086. [http://dx.doi. org/10.1136/jnnp.2005.075887

2. Fletcher JM, Copeland K, Frederick JA, et al. Spinal lesion level in spina bifida: A source of neural and cognitive heterogeneity. J Neurosurg Pediatr 2005;102:268-279. [http://dx.doi.org/10.3171/ ped.2005.102.3.0268]

3. Stevenson KL. Chiari type II malformation: Past, present and future. Neurosurg Focus 2004;16:1-7. [http://dx.doi.org/10.3171/foc.2004.16.2.6]

4. Huber-Okrainec J, Dennis M, Brettschneider J, Spiegler BJ. Neuromotor speech deficits in children and adults with spina bifida and hydrocephalus. Brain and Language 2002;80:592-602. [http://dx.doi. org/10.1006/brln.2001.2620]

5. Fletcher JM, Ostermaier KK, Cirino PT, Dennis M. Neurobehavioral outcomes in spina bifida Processes verus outcomes. J Pediatr Rehabil Med 2008;4:311-324.

6. Dennis M, Barnes MA. The cognitive phenotype of spina bifida meningomyelocoele. Dev Disabil Res Rev 2010;16:31-39. [http://dx.doi.org/10.1002/ddrr.89]

Fletcher JM, Barnes M, Dennis M. Language development in children with spina bifida. Semin Pediatr Neurol 2002;9:201-208. [http://dx.doi.org/10.1053/spen.2002.35500]

8. Barf HA, Verhoef M, Jennekens-Schinkel A, Post MWM, Gooskens RHJM, Prevo AJH. Cognitive status of young adults with spina bifida. Dev Med Child Neurol 2003;45:813-820. [http://dx.doi. org/10.1017/S0012162203001518]

9. Alrikddon-Schmidt AI, Thibadeau JK, Swanson E, et al. The natural history of spina bifida in children pilot project: Research protocol. JMIR Research Protocols 2013;2. 10. Mayes SD, Calhoun SL. Frequency of reading, math, and writing disabilities in children with clinical
disorders. Learning and Individual Differences 2006;16:145-157. [http://dx.doi.org/10.1016/j. disorders. Learning and Individual Differences 2006;16:145-157. [http://dx.doi.org/10.1016/. lindif.2005.07.004

11. Barnes MA, Raghubar KP, English L, Williams JM, Taylor H, Landry S. Longitudinal mediator of achievement in mathematics and reading in typical and atypical development. J Exp Child Psychol 2014;119:1-16. [http://dx.doi.org/10.1016/j.jecp.2013.09.0062014;119:1-16]

12. Kelly NC, Ammerman RY, Rausch JR, et al. Executive functioning and phsyiological adjustment in children and youth with spina bifida. Child Neuropsychol 2012;18:417-431. [http://dx.doi.org/10.10 80/09297049.2011.613814

13. Lomax-Bream LE, Barnes MA, Copeland K, Taylor HB, Landry S. The impact of spina bifida on development across the first 3 years. Dev Neuropsychol 2007;31:1-20. [http://dx.doi.org/10.1207/ s15326942dn3101_1]

14. Swanson ME, Dicianno BE. Psychiatrists and developmental pediatricians working together to improve outcomes in children with spina bifida. Pediatr Clin N Am 2010;57:973-981. [http://dx.doi. org/10.1016/j.pcl.2010.07.016]

15. Burke R, Liptak GS. Providing a primary care medical home for children and youth with spina bifida. Pediatrics 2011;128:2011-2219. [http://dx.doi.org/10.1542/peds.2011-2219] 